\author{
Voix plurielles \\ Volume 3, Numéro 2 : septembre 2006
}

Jean B. Ntakirutimana

\title{
Lecture écocritique de la violence dans Professeur cherche élève ayant désir de sauver le monde de Daniel Quinn
}

Citation MLA : Ntakirutimana, Jean B. «Lecture écocritique de la violence dans Professeur cherche élève ayant désir de sauver le monde de Daniel Quinn .»Voix plurielles 3.2 (septembre 2006). 


\title{
Lecture écocritique de la violence dans Professeur cherche élève ayant désir de sauver le monde de Daniel Quinn
}

\author{
Jean B. Ntakirutimana \\ Université Brock
}

Septembre 2006

$\mathrm{D}$ ans ce roman philosophique, Julie, une préadolescente en mal de vivre, a pour maître à penser Ishmael, un gorille philosophe et télépathe qui, après avoir compris que la cupidité, l'insouciance et la vanité de l'homme moderne sont en train de conduire le monde à sa perte, s'est donné pour mission de recruter des disciples prêts à sauver le monde de sa destruction imminente.

L'enseignement d'Ishmael, basé sur les principes philosophico-pédagogiques de la maïeutique, consiste essentiellement à initier chacun de ses élèves à cette mission salvatrice de haute importance, en leur faisant prendre conscience des grandes menaces qui pèsent sur le monde actuel.

Dans Professeur cherche élève ayant désir de sauver le monde, comme dans l'ensemble de son œuvre, Daniel Quinn insiste sur le fait que tous ces dangers qui menacent le monde moderne tirent leurs origines de la grande révolution qui a vu le jour dans la région $\mathrm{du}$ « ... Croissant fertile, il y a dix mille ans » (98). Ladite révolution a bouleversé les modes de vie des gens, convertissant progressivement les nombreux peuples de chasseurs et de cueilleurs en agriculteurs et éleveurs sédentaires, avec tous les problèmes qui en découlent et qui s'amplifient au jour le jour, comme la destruction des ressources naturelles, la répartition inégale des richesses, les guerres et les multiples formes de violence, le désespoir et l'incertitude du lendemain, l'aliénation par les média et les système éducatifs, etc.

Par la voix d'Ishmael et de Julie, Quinn offre au lecteur une vision manichéenne d'un monde composé de deux groupes aux modes de vies diamétralement opposés, parce que fondés sur des valeurs de bases difficiles à concilier, quasi incompatibles. Il présente, d'une part, le groupe des bons; c'est-à-dire toutes ces communautés qui mènent une vie en harmonie avec la nature, celle des chasseurs-cueilleurs, nomades ou semi-nomades. Quinn appelle les peuples membres de ce groupe «Ceux-qui-laissent » (55) à cause de leurs bonnes habitudes de ne prendre que ce dont ils ont besoin dans l'immédiat, laissant le reste à la nature et à la communauté.

D'autre part, il y a les peuples de mauvais, de dangereux sédentaires, grands destructeurs des 
ressources naturelles et, partant, destructeurs potentiels du monde. Quinn appelle ces peuples « Ceux-qui-prennent » (55) en raison de leur forte tendance à s'accaparer de tout ce qui leur tombe sous la main, ainsi que leur forte propension à accumuler des biens et de se les approprier aux dépens des autres membres du monde vivant.

En plus de vouloir tout s'approprier, Ceux-qui-prennent se considèrent comme les seuls vrais représentants de l'humanité, responsables de sa destiné. « Ils veulent tout gérer, tout organiser, et ne tolèrent pas qu'il puisse se passer dans leur entourage quelque chose qui leur échappe » (104).

Ceux-qui-laissent sont décrits comme des peuples solidaires, sereins et relativement pacifiques. Même les cas de violence vécus par ces communautés ne semblent pas perturber leur harmonie sociale. Consistant en des jeux « d'attaques-surprises » (90), ces cas de violence, malgré leur apparence combative, n'entraînent pas de conséquences néfastes envers la communauté, mais favorisent plutôt la paix.

Cette forme de violence paraît uniquement dictée par les contraintes de survie de l'espèce. Elle est ainsi conçue comme inhérente à toute espèce vivante; et elle est indispensable au maintien de l'équilibre naturel. C'est elle qui « permet à des tribus concurrentes de se maintenir sur un pied d'égalité » (101). En effet, « selon l'esprit tribal, il ne sert à rien de dire aux gens : "On ne doit jamais se battre." Ce qui n'est pas vain, en revanche, c'est de savoir précisément quelle est la meilleure chose à faire lorsqu'il y a eu combat, de sorte que chacun s'en tire sans trop de dommages » (108). Ainsi, la sagesse et le génie de Ceux-qui-laissent créent des mécanismes susceptibles d'endiguer toute manifestation de la violence.

Ceux-qui-prennent, à l'opposé, sont dépeints comme des peuples trop violents, cupides et avides de s'approprier toutes les ressources naturelles. Ils sont donc présentés comme de grands destructeurs de civilisations et du milieu naturel, égoïstes et insatiables dans leurs désirs d'amasser des richesses matérielles. Hypocrites et menteurs, Ceux-qui-prennent ont l'habitude de cacher leurs mauvaises intentions. Ils se montrent toujours sous un jour favorable grâce à une « Mère Culture » (30) omnipotente, capable de dompter même les esprits rebelles, et grâce à un système médiatique aliénant. Les systèmes scolaires qui leur sont sous-jacents sont aussi aliénants, parce qu'inadaptés aux besoins réels du monde en général, et de la jeunesse en particulier.

À l'instar de toute autre forme de bouleversement capital dans les modes de vie, la révolution de Ceux-qui-prennent s'est accompagnée de violences dont les résultats ont engendré encore plus de violences. Que l'on pense aux innombrables guerres, documentées ou non par l'histoire, que les peuples de Ceux-qui-prennent ont lancées contre Ceux-qui-laissent, dans le but de conquérir les territoires de ces derniers, de les dominer et de s'approprier leurs biens. Qu'on s'imagine les multiples révoltes tentées par Ceux-qui-laissent afin de se soustraire à la ferrure des envahisseurs et de recouvrer leur liberté. On n'oubliera pas, non plus, les nombreuses guerres de rivalités que Ceux-qui-prennent ont menées et mènent encore entre eux, souvent pour des motifs farfelus. De nos jours, « le crime est une industrie multimilliardaire, les enfants achètent de la drogue au coin de la rue, et les citoyens poussés à bout passent leur colère en se tirant dessus » (106).

Dans cette œuvre majeure de Daniel Quinn, comme à travers ses autres écrits --tels que The Story of B (Bantam Books, 1996) ${ }^{1}$, Ishmael (Bantam Books, 1992) ${ }^{2}$-- il y aurait probablement lieu d'alléguer que l'auteur brosse un portrait simpliste et naïf du monde ${ }^{3}$. Cependant, à y regarder de près, on se rend compte que Quinn n'a pas tout à fait tort de présenter les choses ainsi, surtout qu'il 
est motivé par des raisons didactiques évidentes. Tout compte fait, il a le mérite d'avoir compris en profondeur les grands dangers qui menacent le monde, et de les présenter d'une manière originale et particulièrement touchante. Quinn a su ausculter et mettre à nu les grands maux des civilisations modernes, lesquelles semblent caractérisées par l'appât du gain, avec toute les formes de violences qui en découlent. Accordons-lui également le crédit d'avoir poussé sa réflexion au-delà d'un simple diagnostic, et de l'avoir complété par des propositions de solutions (206-222) tout aussi originales que l'ensemble de la pensée quinnienne.

\section{Oeuvre}

Quinn, Daniel. Professeur cherche élève ayant désir de sauver le monde. Paris : J'ai lu, 2000. (Traduit de l'anglais étasunien par Valérie Rosier, à partir du titre initial : Quinn, Daniel. My Ishmael. New York : Bantam Books, 1997.)

\section{Notes}

${ }^{1}$ Ouvrage non encore traduit en français.

${ }^{2}$ Quinn, Daniel. Ishmael. Paris : J'ai lu, 1997. (Traduit de l'anglais étasunien par José Malfi)

3 The Story of B (Bantam Books, 1996), Ishmael (Bantam Books, 1992) et Professeur cherche élève ayant désir de sauver le monde (2000) racontent tous la même histoire des enseignements d'Ishmael à ses disciples, histoire consignée de trois façons différentes selon ce que chacun des trois élèves a assimilé de ses entretiens avec Ishmael. En bon pédagogue, ce dernier essaie d'adapter ses leçons à la capacité d'assimilation, et surtout à la personnalité de chaque élève, comme l'explique le maître lui-même en ces termes : « ... aucun élève ne puise la même chose que son voisin. Chacun retient le message à sa façon : il en a reçu une version différente et il transmettra lui-même une version transformée du même message » (70). 